DIW BERLIN

Discussion

Papers
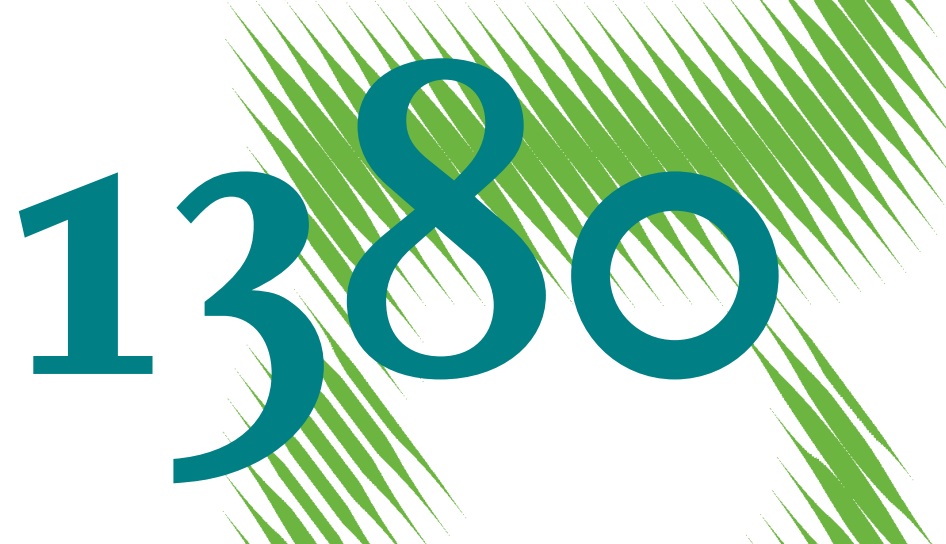

MMI

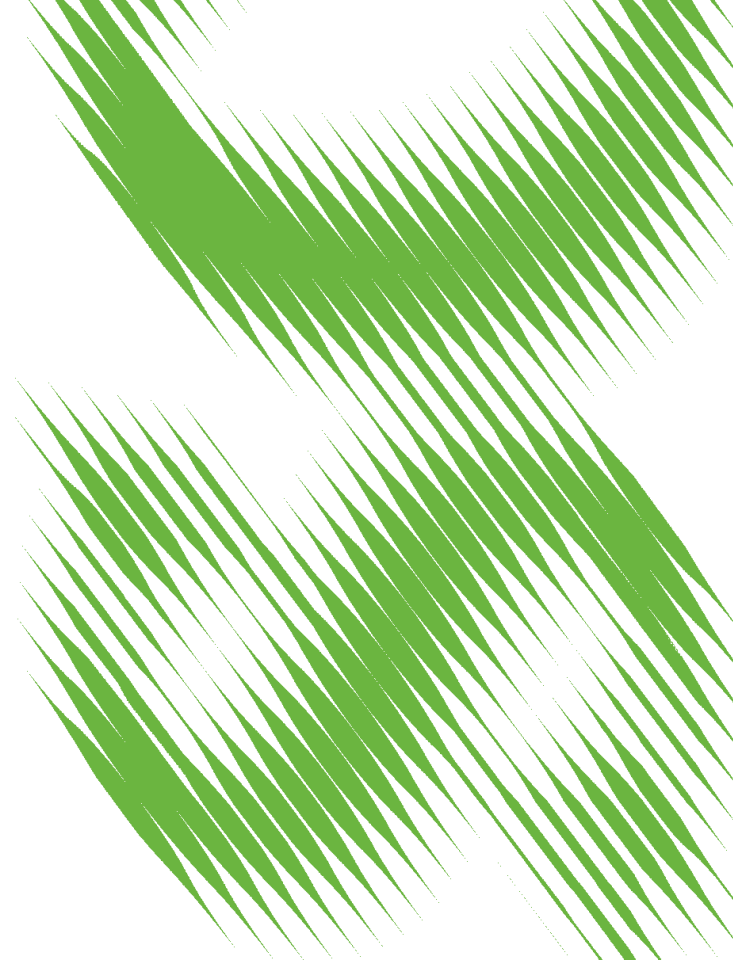

MIMMMMMMMM

Network Expansion to Mitigate Market Power: How Increased Integration Fosters Welfare 
Opinions expressed in this paper are those of the author(s) and do not necessarily reflect views of the institute.

IMPRESSUM

(C) DIW Berlin, 2014

DIW Berlin

German Institute for Economic Research

Mohrenstr. 58

10117 Berlin

Tel. $+49(30) 89789-0$

Fax +49 (30) $89789-200$

http://www.diw.de

ISSN print edition $1433-0210$

ISSN electronic edition 1619-4535

Papers can be downloaded free of charge from the DIW Berlin website:

http://www.diw.de/discussionpapers

Discussion Papers of DIW Berlin are indexed in RePEc and SSRN:

http://ideas.repec.org/s/diw/diwwpp.html

http://www.ssrn.com/link/DIW-Berlin-German-Inst-Econ-Res.html 


\title{
Network expansion to mitigate market power: How increased integration fosters welfare*
}

\author{
Alexander Zerrahn†, Daniel Huppmann \\ German Institute for Economic Research (DIW Berlin) \\ Mohrenstraße 58, 10117 Berlin, Germany \\ azerrahn@diw.de,dhuppmann@diw.de
}

Revised version, August 21, 2014

\begin{abstract}
Lack of transmission capacity hampers the integration of the European electricity market, and thereby precludes reaping the full benefits of competition. In this work, we investigate the extent to which transmission grid expansion promotes competition, efficiency and welfare. To this end, we propose a three-stage model for grid investment: a benevolent planner decides on network upgrades, considering welfare benefits of investments through a reduction of market power exertion by strategic generators. These firms anticipate their impact on market clearing, in particular when lines are congested. In this respect, we provide the first model effectively endogenizing the trade-off between costs of grid investment and benefits from reduced market power potential.

In a three-node network, we illustrate three distinct strategic effects: firstly, by reducing market power exertion, network expansion can promote welfare beyond pure efficiency gains: optimally accounting for strategic generator behavior can push welfare close to a first-best competitive benchmark. Myopically focusing on bottlenecks only can yield suboptimal outcomes. Secondly, network upgrades entail a relative shift of rents from producers to consumers, and thirdly, they may yield suboptimal or even disequilibrium outcomes when strategic behavior of certain market participants is neglected in network investment.
\end{abstract}

Keywords: market power, electricity transmission, network expansion, Generalized Nash equilibrium (GNE), mixed-integer equilibrium problem under equilibrium constraints (MIEPEC)

JEL Codes: L13, L51, C61

\footnotetext{
*The authors would like to thank Friedrich Kunz, Sauleh Siddiqui and Carlos Ruiz for many valuable comments. Financial support by Energie-Control Austria (ECA) for D. Huppmann within the special series "10 years of e-control" is gratefully acknowledged. The views expressed in this article should not be considered as a statement regarding the views of ECA

${ }^{\dagger}$ Corresponding author
} 


\section{Policy: Market Power and Network Expansion}

In the mid-1990s, the European Union started developing the institutional basis for an integrated Internal Energy Market (IEM): formerly state-owned utilities were unbundled and the electricity transmission grid was opened up to new generators entering the power market. At the same time, regional energy exchanges were established in order to provide market places with transparent wholesale prices.

The formation of the Council of European Energy Regulators (CEER) in 2000 and the Agency for the Cooperation of Energy Regulators (ACER) in 2010 laid institutional cornerstones for developing a level playing field. Nevertheless, market concentration remains high: in ten Member States, the largest generating company has a market share above $70 \%$ (European Commission, 2012, data for 2010). By means of further European integration, this high degree of concentration could be reduced, thereby mitigating the potential to exert market power while consequently enhancing efficiency and increasing welfare.

Interconnectors between countries, however, were originally constructed for contingencies, not to facilitate cross-border trade. What is missing, thus, are sufficient physical interconnector capacities to accomplish a fully integrated market. Strong indicators for incomplete integration in Europe are found through empirical analyses which detect persistent wholesale price spreads between countries (Zachmann, 2008, ACER/CEER, 2013, Böckers, Haucap, and Heimeshoff, 2013). This trend coincides with an increasing use of commercial transfer capacities and a declining number of flows against the price differential, pointing to a more efficient use of interconnector capacity. These findings, thus, lend stark evidence that, firstly, physical capacities are still insufficient to achieve full integration, and, secondly, inefficiencies in the allocation mechanism are not the principle drivers of this incomplete convergence since the introduction of market coupling.

The economics of European electricity market integration has various dimensions. Beyond efficiency gains from trade, more efficient feed-in of variable renewables as well as enhanced security of supply, integration is essential to ensure vigorous inter-regional competition where concentration on single markets remains high - this aspect is the focus of our contribution.

While we motivate our research by the institutional setting in Europe, the underlying mechanisms are present in other markets, in particular the US. Although featuring different market designs, the federal structure with state-wide or regional systems can be seen as an analogue to European countries with constrained interconnection and potentially dominant generators in local markets.

Modeling the interaction between firms that strategically decide on output levels and investment in networks is still challenging - especially to properly account for gaming opportunities between generators and network operators. In this paper, we investigate to what extent transmission grid expansion between regional markets offers a way to realize welfare improvements beyond efficiency gains; that is welfare gains due to a reduction of market power exertion. The underlying mechanism is the following: in case transmission capacities are scarce, regional markets are separated and incumbent firms face little outside competitive pressure. Moreover, they also might find it profitable to aggressively congest lines to adjacent regions in order to prevent competitors from entering their domestic market. These implications of low network capacities hampering competition can be mitigated by means of transmission grid expansion.

The major theoretical contribution of our work consists of endogenously including the trade-off between costs and benefits of network expansion into one integrated model. In a three-stage game, each agent anticipates the impact of her actions on subsequent decisions: the transmission system planner accounts for their effect on strategic firms' behavior, and those, in turn, anticipate their impact on the system operator clearing the market. Thereby, we endogenize transmission grid expansion decisions and do not have to rely on the exogenous variation of parameters or the analysis of scenarios. To the best of our knowledge, this is 
the first contribution explicitly incorporating this trade-off. Moreover, we apply a recent method for solving Equilibrium Problems under Equilibrium Constraints (EPEC) and extend it mathematically as well as with respect to the interpretation of the players.

Applying our model to a sample network, we identify three distinct effects: firstly, network expansion can increase welfare by mitigating market power potential - leading to a second-best equilibrium close to the first-best outcome of perfectly competitive markets. In some cases, a relatively "thin" line can be sufficient to enforce a higher degree of competition. Moreover, expansion of lines may be required to prohibit gaming - even if these lines are not congested in equilibrium. In this vein, only focusing on existing bottlenecks may yield suboptimal outcomes. Secondly, beyond an increase in welfare, optimal network expansion entails a relative shift of rents from producers to consumers. Due to the prevention of strategically exploiting bottlenecks, excessive rent extraction by generators can be mitigated such that consumers benefit. Thirdly, ignoring strategic behavior by firms may yield suboptimal decisions for network upgrades - and may possibly create situations in which no stable equilibria can be attained.

The remainder of this paper is structured as follows: Section 2 gives an overview of the existing literature and relates it to our research, Section 3 describes our modeling approach. The mathematical formulation is explained in detail in Section 4 , and Section 5 discusses numerical results. Section 6 concludes and outlines avenues for further research.

\section{Theory \& Literature: Modeling Market Power in Electricity Networks}

The analysis of strategic generator behavior in networks has been in the focus of the academic community for several years. Neuhoff et al. (2005) point out that the "devil is in the details": comparing different approaches concerning the treatment of transmission constraints in two-stage models, the authors identify the fundamental challenges in rendering a realistic representation of interactions between strategic generation and clearing of multiple markets. Specifically, there are two methods to incorporate the transmission system operators' (TSO) optimization programs (cf. Hobbs, Rijkers, and Boots, 2005), depending on whether strategic players anticipate their impact on network operation (Stackelberg) or not (naïve). The latter perspective considerably reduces model complexity at the cost of excluding strategic effects between dominant firms and network operation. Examples comprise the exogenous assumption of rationing mechanisms in case transmission capacity is scarce (Willems, 2002), shifting of an inelastic demand parameter (Boffa, Pingali, and Vanconi, 2010), and strategic players treating transmission charges arising from TSO optimization as exogenous in their constraint sets (Tanaka, 2009). Hobbs and Rijkers (2004) choose an in-between approach where generators hold conjectures concerning transmission price responses. The Stackelberg assumption is pursued in Cunningham, Baldick, and Baughman (2002) and Ehrenmann and Neuhoff (2009), who explicitly derive reaction functions and closed-form solutions under rigid assumptions for some special cases. Alternatively, both Hobbs, Metzler, and Pang (2000) and Ehrenmann and Neuhoff (2009) propose algorithmic solutions based on diagonalization methods. For all of these approaches, however, network expansion remains exogenous to the model and is restricted to a limited number of cases in varying line constraint parameters.

\section{Theoretical and empirical research}

For a reduced special case, the assumption of strategic firms anticipating their impact on the network situation was picked up in the theoretical industrial organization literature: in their seminal contribution, sometimes referred to as the thin-line paper, Borenstein, Bushnell, and Stoft (2000) demonstrate that in a simple two-node network even a line with relatively low capacity may be enough to foster competition and evoke substantial welfare gains. In 
this context, it is irrelevant whether the line is actually utilized as long as its capacity is sufficiently high to prevent strategic generators from deliberately congesting the grid.

The literature also features contributions with a distinct theoretical industrial organization (Ruderer and Zöttl, 2012; Léautier, 2013) or empirical focus (Gebhardt and Höffler, 2013 Ryan, 2013; Wolak, 2012). Both streams provide evidence that network expansion is a useful approach to mitigate market power potential.

\section{Three-stage models}

The modeling stream most similar to the work presented here was introduced by Sauma and Oren (2006): under the label proactive planning, they present a methodology to evaluate network expansion projects such that their effect on strategic players is taken into account. Variations and extensions of this paper are developed by Pozo, Contreras, and Sauma (2013a) and Pozo, Sauma, and Contreras (2013b). They propose an improved solution technique allowing for more flexibility concerning line expansions. The results of all three analyses are in line with intuition: network expansion has the potential to enhance social welfare, and this increase is greater when the network planning entity proactively takes strategic behavior by market participants into account. Although the studies are based on three-stage models and incorporate strategic interactions among generating firms, we depart from their approach in two fundamental ways:

Firstly, by employing results from duality theory following Ruiz, Conejo, and Smeers (2012), we are able to reformulate the market model such that first order optimality conditions can be explicitly derived. The virtue of this methodological contribution lies in circumventing the inconvenient usage of iterative algorithms or discretization of decision variables. Thereby, we are not restricted to evaluating a small number of predefined cases as usually done in the analysis of network expansion options, but can endogenously trade off the costs and benefits of line upgrades.

Secondly, we do not consider investments in generation capacity while inelastic demand is satisfied by a perfectly competitive dispatch, as in Pozo, Contreras, and Sauma (2013a) and Pozo, Sauma, and Contreras (2013b). Instead, we analyze strategic interaction on a spot market with existing capacities: in Sauma and Oren (2006), and Pozo, Contreras, and Sauma (2013a) there is only one generation technology whose marginal production costs decrease in capacity invested, an assumption needed to justify investment activities by firms. On the one hand, we do not find the assumption of lower marginal costs due to a "larger" generating unit convincing. On the other hand, we believe that factors other than the potential exertion of market power due to limited network capacities, which are not captured by a model of this kind, also drive the decisions to invest in certain technologies. Among these are predominantly the temporal patterns of demand and renewables, but also the existing fleet of a firm, aspects of financing, fuel prices, or innovation processes. Léautier (2013) and, in a more simplified form, Zöttl (2011), show that the analysis of strategic investment behavior, even with only two distinct technologies (base and peak), is already complicated. Results are substantially related to assumptions on expectations about the occurrence of different spot market demand scenarios or the policy framework.

Summing up, if the potential to exert market power with an existing power plant park does exist, strategic firms will have the incentive to exploit it. Therefore, we focus on the analysis of strategic spot market behavior under endogenous network configurations.

\section{Application: A Three-Stage Model}

We propose a three-stage model in which a benevolent social planner anticipates the equilibria among strategic generators, which are potentially altered due to transmission grid 
Table 1: Model structure.

\begin{tabular}{|c|c|c|c|}
\hline Level & Time & Player(s) and decisions & Objectives \\
\hline $\mathrm{I}$ & $\begin{array}{l}\text { Network } \\
\text { expansion }\end{array}$ & $\begin{array}{l}\text { Benevolent planner } \\
\text { transmission capacity investment }\end{array}$ & $\begin{array}{l}\text { maximize welfare } \\
\text { less investment costs }\end{array}$ \\
\hline II & & $\begin{array}{l}\text { Strategic Generators } \\
\text { generation at each node }\end{array}$ & maximize profit \\
\hline III & Spot market & $\begin{array}{l}\text { Independent System Operator (ISO) } \\
\text { dispatch of competitive generation, } \\
\text { load, nodal prices, network flows } \\
\text { subject to network feasibility }\end{array}$ & $\begin{array}{l}\text { maximize welfare } \\
\text { (short-run) }\end{array}$ \\
\hline
\end{tabular}

expansion 11 Table 1 illustrates the model structure, involved players and their decisions. In the following, we explain the stages bottom-up according to the principle of backward induction and leave the mathematical formulation for the next Section 4.

\section{The spot market}

In the third stage, an Independent System Operator (ISO) dispatches competitive fringe plants and ensures feasible network flows. Taking strategic firms' generation and network capacity as given, she assigns locational prices to each node in the system, such that markets clear and network flows are feasible. As is standard in the literature, we employ a lossless direct current (DC) load flow approximation explicitly capturing loop flows in meshed networks (Schweppe et al., 1988). In the second model stage, the strategic producers, in turn, anticipate the effect of their generation decisions on the ISO and nodal prices. In particular, they can potentially generate excess returns by appropriating rents through congesting the network. Amongst each other, the strategic generators play a Nash-Cournot game; that is, they anticipate the ISO's reaction in the third stage, while taking the generation levels of other strategic firms and the first-stage network expansion decisions as given. By withholding capacity or deliberately causing network congestion, they are able to increase prices above the competitive level.

In our paper, we restrict ourselves to analyzing (Cournot) quantity strategies for firms: firstly, they constitute an established and convenient workhorse model (cf. Limpaitoon, Chen, and Oren, 2014), and quantity games were found to capture prevailing data for representing short-run competition on the electricity spot market as well as more involved supply function equilibrium concepts featuring price-quantity strategies (Willems, Rumiantseva, and Weigt, 2009). Secondly, this approach allows us to compare our results to findings from the theoretical literature (in particular the aforementioned article by Borenstein, Bushnell, and Stoft, 2000). In this work, we abstain from introducing mixed strategies although those might deliver theoretical existence results in case pure strategy equilibria fail to exist. We are, however, not convinced that randomization over quantities provides a solid basis for understanding electricity markets.

The spot market, therefore, constitutes a two-stage game, with strategic firms on the "upper level" (II) and the ISO representing the "lower level" (III). We use the notation of an ISO for convenience, but it is equivalent to the equilibrium in an electricity market

\footnotetext{
${ }^{1}$ In a similar three-stage setup, Huppmann and Egerer (2014) analyze the dimension of national-strategic behavior: they assume a competitive spot market in the third stage, but national entities strategically deciding on network expansion to maximize national welfare in the second stage. The first stage is constituted by a supra-national planner deciding on cross-border interconnector investments. Their model is solved applying the same methodology of reformulating the third-stage constraints as presented here.
} 
that is perfectly competitive except for the presence of strategic generators. It is furthermore equivalent to the coupling of nodal markets in the most efficient way. Actual market coupling procedures are, in some European regions, based on so-called Net Transfer Capacities (NTC), in which commercial flows may underutilize or overstress existing capacities. In reality, plants are redispatched after the market clearing process to ensure network feasibility. In our approach, flows are directed in a welfare-optimal manner, hence we directly render an upper bound for efficiency and welfare $2^{2}$

\section{Network expansion}

In the first stage of our model, a benevolent social planner maximizes total welfare by deciding upon the level of transmission grid expansion. While anticipating how changes in the network topology will influence the Nash equilibrium outcome on the spot market, she faces a trade-off between the costs of grid expansion and the welfare-enhancing effects of integration between different nodes: on the one hand, efficiency gains due to a reduction in congestion and access to less expensive generation capacity; on the other hand, increasing competition among the strategic generators weakens their potentially dominant positions and thus reduces the potential to exert market power.

\section{Mathematical Formulation}

In the following section, we present our model from a mathematical point of view and explain how we overcome methodologically challenging issues. Throughout the analysis, we assume a system with welfare-optimal nodal prices and power flows accomplished by the ISO, given the quantities sold by the strategic generators and network capacity, which includes the line expansion by the benevolent network planner.

\section{Stage III: A competitive market - or the ISO}

In the third model stage, the ISO maximizes welfare $1 \mathrm{a}$ - the sum of consumer surplus, generator profit and congestion rent, minus variable production costs of utilized plants - over the dispatch of non-strategic fringe plants at all nodes $n$, consumed quantities $d_{n}$ and voltage angles $\delta_{n}$, while ensuring feasible network flows. We employ a lossless DC load flow (DCLF) approximation incorporating loop flows by voltage angles at each node (Schweppe et al. 1988 - based on $H$ and $B$ matrices, which is equivalent to a power transmission distribution factor (PTDF) formulation (for a detailed exposition, see for example Leuthold, Weigt, and v. Hirschhausen, 2012). The constraint set consists of the nodal electricity balances (1b), feasibility of flows on each line $\sqrt{1 \mathrm{c}}-\sqrt{1 \mathrm{~d}})$ as well as maximum generation $(1 \mathrm{e})$ and nonnegativity restrictions $\sqrt{1 \mathrm{f}}-\mathrm{1g}$ ), each with its respective dual variable given in parentheses. The inverse nodal demand function is assumed linear with reservation price $a_{n}$ and slope parameter $b_{n}$. Individual plants are denoted by index $s$, where each plant is exogenously mapped to a node $n$. An additional superscript $F$ denotes that the respective plant belongs to the fringe supply dispatched at marginal cost by the ISO. All other plants, indicated by superscript $S$, are owned by strategic generators - therefore, their generation is treated as exogenous by the ISO. Generation quantities for each plant $s$ are given by $g_{s}^{S}$ or $g_{s}^{F}$, respectively. From the ISO's point of view, line expansion $e_{l}$ is an exogenous parameter. For notational convenience, we introduce set $\mathfrak{S}_{n}$ containing all plants at node $n$, assemble all fringe plants in $\mathfrak{F}$, and all nodes at which non-zero demand is located in $\mathfrak{N}$. The optimization

\footnotetext{
${ }^{2}$ In an NTC system, a fixed calculatory amount of interconnector capacity between two countries is quoted in advance, and the cheapest bids from one market to another are accepted until this capacity limit is reached. Resulting physical power flows, however, may diverge from merchant flows due to alternate current physical characteristics (Kirchhoff's laws). See Oggioni et al. (2012) for an analysis of this issue.
} 
problem reads as follows:

$$
\begin{aligned}
\min _{d, \delta, g^{F}}-\sum_{n \in \mathfrak{N}}\left[\left(a_{n}-\frac{1}{2} b_{n} d_{n}\right) d_{n}\right]+\sum_{s \in \mathfrak{F}} c_{s}^{G} g_{s}^{F} & \\
\text { s.t. } \quad-\sum_{s \in \mathfrak{S}_{n}}\left(g_{s}^{S}+g_{s}^{F}\right)+\sum_{k} B_{n k} \delta_{k}+d_{n}=0 & \left(p_{n}\right) \quad \forall n \\
-\bar{f}_{l}-e_{l}+\sum_{k} H_{l k} \delta_{k} \leq 0 & \left(\bar{\mu}_{l}\right) \quad \forall l \\
-\bar{f}_{l}-e_{l}-\sum_{k} H_{l k} \delta_{k} \leq 0 & \left(\underline{\mu}_{l}\right) \quad \forall l \\
-\bar{g}_{s}^{F}+g_{s}^{F} \leq 0 & \left(\beta_{s}\right) \quad \forall s \in \mathfrak{F} \\
-g_{s}^{F} \leq 0 & \left(\psi_{s}\right) \quad \forall s \in \mathfrak{F} \\
-d_{n} \leq 0 & \left(\phi_{n}\right) \quad \forall n \in \mathfrak{N} \\
\delta_{\hat{n}}=0 & (\gamma)
\end{aligned}
$$

The hub node of the network is given by $\hat{n}(1 \mathrm{~h})$. In case there is no demand at node $n$, we fix $d_{n}$ and $\phi_{n}$ at zero. Differentiating yields the respective first order KKT conditions:

$$
\begin{aligned}
& 0=c_{s}^{G}-p_{n, s \in \mathfrak{G}_{n}}+\beta_{s}-\psi_{s} \quad, \quad g_{s}^{F}(\text { free }) \quad \forall s \in \mathfrak{F} \\
& 0=-a_{n}+b_{n} d_{n}+p_{n}-\phi_{n} \quad, \quad d_{n} \text { (free) } \quad \forall n \in \mathfrak{N} \\
& 0=\sum_{k} B_{k n} p_{k}+\sum_{l} H_{l n}\left(\bar{\mu}_{l}-\underline{\mu}_{l}\right)+\left\{\begin{array}{ll}
\gamma & \text { if } n=\hat{n} \\
0 & \text { else }
\end{array}\right\} \quad, \quad \delta_{n} \text { (free) } \quad \forall n \\
& 0=\sum_{s \in \mathfrak{G}_{n}}\left(g_{s}^{S}+g_{s}^{F}\right)-\sum_{k} B_{n k} \delta_{k}-d_{n} \quad, \quad p_{n} \text { (free) } \quad \forall n \\
& 0 \leq \bar{f}_{l}+e_{l}-\sum_{k} H_{l k} \delta_{k} \quad \perp \quad \bar{\mu}_{l} \geq 0 \quad \forall l \\
& 0 \leq-\bar{f}_{l}-e_{l}-\sum_{k} H_{l k} \delta_{k} \quad \perp \quad \underline{\mu}_{l} \geq 0 \quad \forall l \\
& 0 \leq \bar{g}_{s}^{F}-g_{s}^{F} \quad \perp \quad \beta_{s} \geq 0 \quad \forall s \in \mathfrak{F} \\
& 0 \leq g_{s}^{F} \quad \perp \quad \psi_{s} \geq 0 \quad \forall s \in \mathfrak{F} \\
& 0 \leq d_{n} \quad \perp \quad \phi_{n} \geq 0 \quad \forall n \in \mathfrak{N} \\
& 0=\delta_{\hat{n}} \quad, \quad \gamma(\text { free })
\end{aligned}
$$

Optimality conditions 2a - 2j] will be incorporated as constraints in the higher stages of our model. However, in standard complementarity form, they are bilinear and contain an either-or requirement, the complementarity condition. Without reformulation, it is challenging to include the lower-level optimization problem in the upper-level problem.

For resolving this issue, previous research provides three options: firstly, the approach of a disjunctive constraints reformulation (Fortuny-Amat and McCarl, 1981), expressing the complementarity constraints with help of binary variables. This is, for example, used by Gabriel and Leuthold (2010). Secondly, Siddiqui and Gabriel (2013) propose a reformulation of the bilinearities using Schur's decomposition and so-called SOS1-type variables. Thirdly, Ruiz, Conejo, and Smeers (2012) use a reformulation of lower-level optimality based on strong duality.

The first and second method come with the drawback of introducing additional constraints and binary variables, which complicates the analysis computationally. Most importantly, however, we then would be left with a discretely-constrained Nash-Cournot game. Here, it is again not possible to derive KKT conditions without further modifications. Although there has been progress in that field - Gabriel et al. (2013) propose a method to 
tackle that issue by relaxing either integrality or complementarity - theoretical research is still ongoing and does not provide a ready-made toolbox.

We therefore pursue the third option: to solve for the Nash equilibrium of the overall three stage game, we follow the methodology proposed by Ruiz, Conejo, and Smeers (2012) and reformulate the ISO's optimization problem in the lowest stage (III) using strong duality. By employing an elastic demand curve - an important assumption when it comes to analyzing market power - we extend their approach from the purely linear case, as the ISO problem is quadratic in our model. The reformulated third stage optimality constraints then provide the feasible space for the second stage strategic generators' Nash-Cournot game.

Generally, the Lagrange dual function is a value function taking the dual variables of the original primal problem as arguments. It is defined as the infimum of the Lagrange primal function over the primal decision variables. The Lagrange dual problem then consists of finding the dual variables that maximize the Lagrangian dual function - that is in detecting the greatest infimum of the primal problem over all dual variables. Reformulating primal Problem 1 accordingly, the ISO minimization of negative welfare, straightforward algebra yields the dual problem for our case:

$$
\begin{aligned}
\max _{p, \bar{\mu}, \underline{\mu}, \beta, \phi, \psi, \gamma}-\frac{1}{2} \sum_{n \in \mathfrak{N}} \frac{1}{b_{n}}\left[a_{n}-p_{n}+\phi_{n}\right]^{2} & -\sum_{l}\left(\bar{f}_{l}+e_{l}\right)\left(\bar{\mu}_{l}+\underline{\mu}_{l}\right) \\
& -\sum_{n} p_{n}\left(\sum_{s \in \mathfrak{S}_{n}} g_{s}^{S}\right)-\sum_{s \in \mathfrak{F}} \beta_{s} \bar{g}_{s}^{F}
\end{aligned}
$$

s.t. 2a $, 2 \mathrm{~b}, 2 \mathrm{c}$ without complementarity,

$$
\bar{\mu}, \underline{\mu}, \phi, \beta, \psi \geq 0, p, \gamma \in \mathbb{R}
$$

By definition, the optimal value of the dual problem is no larger than the optimal value of the primal problem in any case. The difference between these two values is called duality gap. Under certain conditions, the duality gap collapses to zero - a property referred to as strong duality. Because the primal's objective is convex and all constraints are linear, strong duality holds in our setup. The optimal values of the primal function and the dual function, thus, are identical. For the same reasons, KKT conditions of the ISO problem are both necessary and sufficient, given the network and decisions of the strategic generators.

Therefore, both the KKT conditions and the identity of the primal and dual functions are equivalent descriptions of a global solution of the ISO welfare maximization problem. Replacing the former by the latter, hence, leaves us with a representation of optimality on stage three without explicitly incorporating mathematically inconvenient complementarity conditions. Instead of $(2 \mathrm{a})-(2 \mathrm{j})$, we utilize:

$$
\begin{gathered}
-\sum_{n \in \mathfrak{N}}\left[\left(a_{n}-\frac{1}{2} b_{n} d_{n}\right) d_{n}\right]+\sum_{s \in \mathfrak{F}} c_{s}^{G} g_{s}^{F}+\frac{1}{2} \sum_{n \in \mathfrak{N}} \frac{1}{b_{n}}\left[a_{n}-p_{n}+\phi_{n}\right]^{2}+ \\
\quad+\sum_{n} p_{n}\left(\sum_{s \in \mathfrak{S}_{n}} g_{s}^{S}\right)+\sum_{l}\left(\bar{f}_{l}+e_{l}\right)\left(\overline{\mu_{l}}+\underline{\mu_{l}}\right)+\sum_{s \in \mathfrak{F}} \beta_{s} \bar{g}_{s}^{F} \leq 0
\end{gathered}
$$

Constraint 4a ensures feasibility for both the primal and dual problem; 4b imposes a zero duality gap and, as a consequence, an optimal solution to both the primal and dual problem. Note that we do not state an equality here. By definition, the primal objective is weakly greater than the dual objective value. Requiring the reverse inequality to hold imposes identity.

\section{Stage II: Strategic firms}

Now, we introduce the second model stage: strategic firms own plants $s$ at nodes $n$. Let $\mathfrak{S}_{i}$ denote the set of all plants owned by strategic firm $i$, while $g_{i}^{S}$ is the respective production 
vector, and $\mathfrak{D}^{3}$ the set of Lagrange multipliers from the third-stage ISO problem. Firm $i$ 's minimization task thus reads:

$$
\min _{g_{i}^{S}, g^{F}, \delta, d, \mathfrak{D}^{3}}-\sum_{n} \sum_{s \in\left(\mathfrak{S}_{n} \cap \mathfrak{S}_{i}\right)}\left(p_{n}-c_{s}^{G}\right) g_{s}^{S}
$$

where each firm $i$ faces the following constraints:

$$
\begin{array}{rll}
-\bar{g}_{s}^{S}+g_{s}^{S} \leq 0 & \left(\beta_{s}^{S}\right) \quad \forall s \in \mathfrak{S}_{i} \\
-g_{s}^{S} \leq 0 & \left(\psi_{s}^{S}\right) & \forall s \in \mathfrak{S}_{i}
\end{array}
$$

and the constraints arising from the ISO problem:

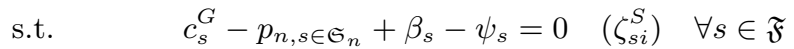

$$
\begin{aligned}
& -a_{n}+b_{n} d_{n}+p_{n}-\phi_{n}=0 \quad\left(\eta_{n i}^{S}\right) \quad \forall n \in \mathfrak{N} \\
& \sum_{k} B_{k n} p_{k}+\sum_{l} H_{l n}\left(\bar{\mu}_{l}-\underline{\mu}_{l}\right)+\left\{\begin{array}{ll}
\gamma & \text { if } n=\hat{n} \\
0 & \text { else }
\end{array}\right\}=0 \quad\left(\theta_{n i}^{S}\right) \quad \forall n \\
& -\sum_{s \in \mathfrak{S}_{n}}\left(g_{s}^{S}+g_{s}^{F}\right)+\sum_{k} B_{n k} \delta_{k}+d_{n}=0 \quad\left(\iota_{n i}^{S}\right) \quad \forall n \\
& -\bar{f}_{l}-e_{l}+\sum_{k} H_{l k} \delta_{k} \leq 0 \quad\left(\bar{\mu}_{l i}^{S}\right) \quad \forall l \\
& -\bar{f}_{l}-e_{l}-\sum_{k} H_{l k} \delta_{k} \leq 0 \quad\left(\underline{\mu}_{l i}^{S}\right) \quad \forall l \\
& -\bar{g}_{s}^{F}+g_{s}^{F} \leq 0 \quad\left(\beta_{s i}^{S F}\right) \quad \forall s \in \mathfrak{F} \\
& -g_{s}^{F} \leq 0 \quad\left(\psi_{s i}^{S F}\right) \quad \forall s \in \mathfrak{F} \\
& -d_{n} \leq 0 \quad\left(\phi_{n i}^{S}\right) \quad \forall n \in \mathfrak{N} \\
& \delta_{\hat{n}}=0 \quad\left(\gamma_{i}^{S}\right) \\
& -\sum_{n \in \mathfrak{N}}\left[\left(a_{n}-\frac{1}{2} b_{n} d_{n}\right) d_{n}\right]+\sum_{s \in \mathfrak{F}} c_{s}^{G} g_{s}^{F}+\frac{1}{2} \sum_{n \in \mathfrak{N}} \frac{1}{b_{n}}\left[a_{n}-p_{n}+\phi_{n}\right]^{2}+ \\
& +\sum_{n} p_{n}\left(\sum_{s \in \mathfrak{S}_{n}} g_{s}^{S}\right)+\sum_{l}\left(\bar{f}_{l}+e_{l}\right)\left(\overline{\mu_{l}}+\underline{\mu_{l}}\right)+\sum_{s \in \mathfrak{F}} \beta_{s} \bar{g}_{s}^{F} \leq 0 \quad\left(\xi_{i}^{S}\right)
\end{aligned}
$$

As constraints (5d) - (5n) describe the optimality conditions of the ISO on the lower stage III, firm $i$ faces a Mathematical Program under Equilibrium Constraints (MPEC). Combining those MPECs for all firms to find a Nash equilibrium between them yields an Equilibrium Problem under Equilibrium Constraints (EPEC). Due to our duality reformulation of the third-stage optimality constraints, as derived in the previous section, we resolve difficulties incurred within this type of model; the optimality conditions of the ISO are already incorporated in the constraint set. Two caveats, however, remain.

Firstly, observe that constraints $5 \mathrm{~d}$ - $-5 \mathrm{n}$ ) arising from the ISO problem are identical for each player whereas, in principle, their valuation may differ across players - the Lagrange multipliers are specific to each firm $i$. Formally, this constitutes a Generalized Nash Equilibrium (GNE) due to the shared constraints. Therefore, there are more endogenous variables than distinct equations. Mathematically, this yields an under-determined system of equations; the implied degrees of freedom potentially admit a multitude of solutions. As a remedy, Harker (1991) proposes assigning identical multipliers across players. Implicitly, this assumes an underlying coordination mechanism like an auction toward the valuation of shared limiting factors. Alternatively, fixed ratios between multipliers are proposed by Oggioni et al. (2012), or a multiplicative decomposition into a common endogenous and a fixed exogenous part (Kunz and Zerrahn, 2013). All these remedies, however, require exogenous assumptions about some form of coordination between players, which we do not see 
sustained here. Instead, we tackle this issue by a disjunctive constraints reformulation as described below.

As a second caveat, the objective function $(5 \mathrm{c})$ and the constraint $(5 \mathrm{n})$ are non-convex such that KKT points of the MPEC are neither necessary nor sufficient for optimality. Moreover, as in any EPEC-type problem, there may exist multiple Nash equilibria - as demonstrated by Borenstein, Bushnell, and Stoft (2000) for strategic electricity generators in constraining networks. We return to both issues below and first derive the KKT conditions for all firms.

$$
\begin{aligned}
& 0=-p_{n, s \in \mathfrak{G}_{n}}+c_{s}^{G}+\beta_{s}^{S}-\psi_{s}^{S}-\iota_{n i, s \in \mathfrak{G}_{n}, s \in \mathfrak{G}_{i}}^{S}+\xi_{i, s \in \mathfrak{G}_{i}}^{S} p_{n, s \in \mathfrak{G}_{n}}, g_{s}^{S} \quad \text { (free) } \quad \forall s \notin \mathfrak{F} \quad \text { (6a) } \\
& 0=-\iota_{n i, s \in \mathfrak{S}_{n}}^{S}+\beta_{s i}^{S F}-\psi_{s i}^{S F}+\xi_{i}^{S}\left(c_{s}^{G}+\beta_{s}\right), g_{s}^{F} \quad \text { (free) } \quad \forall s \in \mathfrak{F}, i(6 \mathrm{~b}) \\
& 0=-\sum_{s \in\left(\mathfrak{S}_{n} \cap \mathfrak{S}_{i}\right)} g_{s}^{S}-\sum_{s \in\left(\mathfrak{S}_{n} \cap \mathfrak{F}\right)} \zeta_{s i}^{S}+\eta_{n i}^{S}+\sum_{k} \theta_{k i}^{S} B_{n k}+ \\
& +\xi_{i}^{S} \frac{1}{b_{n}}\left(p_{n}-a_{n}-\phi_{n}\right)+\xi_{i}^{S} \sum_{s \in \mathfrak{G}_{n}} g_{s}^{S}, p_{n} \quad \text { (free) } \quad \forall n, i \quad(6 \mathrm{c}) \\
& 0=b_{n} \eta_{n i}^{S}+\iota_{n i}^{S}-\phi_{n i}^{S}-\xi_{i}^{S}\left(a_{n}-b_{n} d_{n}\right), d_{n} \quad \text { (free) } \quad \forall n, i \\
& 0=\sum_{k} B_{k n} \iota_{k i}^{S}+\sum_{l} H_{l n}\left(\bar{\mu}_{l i}^{S}-\underline{\mu}_{l i}^{S}\right)+\left\{\begin{aligned}
\gamma_{i}^{S} & \text { if } n=\hat{n} \\
0 & \text { else }
\end{aligned}\right\}, \delta_{n} \text { (free) } \forall n, i \\
& 0 \leq \zeta_{s i}^{S}+\xi_{i}^{S} \bar{g}_{s}^{F} \perp \beta_{s} \geq 0 \quad \forall s \in \mathfrak{F}, i \\
& 0 \leq-\zeta_{s i}^{S} \perp \psi_{s} \geq 0 \quad \forall s \in \mathfrak{F}, i \\
& 0 \leq-\eta_{n i}^{S}+\xi_{i}^{S}\left[\frac{1}{b_{n}}\left(\phi_{n}+a_{n}-p_{n}\right)\right] \perp \phi_{n} \geq 0 \quad \forall n, i \\
& 0 \leq \sum_{n} \theta_{n i}^{S} H_{l n}+\xi_{i}^{S}\left(\bar{f}_{l}+e_{l}\right) \perp \bar{\mu}_{l} \geq 0 \quad \forall l, i \\
& 0 \leq-\sum_{n} \theta_{n i}^{S} H_{l n}+\xi_{i}^{S}\left(\bar{f}_{l}+e_{l}\right) \perp \underline{\mu}_{l} \geq 0 \quad \forall l, i \\
& 0=\theta_{\hat{n} i}^{S}, \gamma \quad(\text { free }) \quad \forall i \\
& 0 \leq \bar{g}_{s}^{S}-g_{s}^{S} \perp \beta_{s}^{S} \geq 0 \quad \forall s \notin \mathfrak{F} \\
& 0 \leq g_{s}^{S} \perp \psi_{s}^{S} \geq 0 \quad \forall s \notin \mathfrak{F} \\
& 0=c_{s}^{G}-p_{n, s \in \mathfrak{G}_{n}}+\beta_{s}-\psi_{s}, \zeta_{s i}^{S} \quad(\text { free }) \quad \forall s \in \mathfrak{F}, i(6 \mathrm{n}) \\
& 0=-a_{n}+b_{n} d_{n}+p_{n}-\phi_{n}, \eta_{n i}^{S} \quad(\text { free }) \quad \forall n \in \mathfrak{N}, i \\
& 0=\sum_{k} B_{k n} p_{k}+\sum_{l} H_{l n}\left(\bar{\mu}_{l}-\underline{\mu}_{l}\right)+\left\{\begin{array}{ll}
\gamma & \text { if } n=\hat{n} \\
0 & \text { else }
\end{array}\right\}, \theta_{n i}^{S} \quad \text { (free) } \quad \forall n, i \\
& 0=-\sum_{s \in \mathfrak{S}_{n}}\left(g_{s}^{S}+g_{s}^{F}\right)+\sum_{k} B_{n k} \delta_{k}+d_{n}, \iota_{n i}^{S} \quad \text { (free) } \quad \forall n, i \\
& 0 \leq \bar{f}_{l}+e_{l}-\sum_{k} H_{l k} \delta_{k} \perp \bar{\mu}_{l i}^{S} \geq 0 \quad \forall l, i \\
& 0 \leq \bar{f}_{l}+e_{l}+\sum_{k} H_{l k} \delta_{k} \perp \underline{\mu}_{l i}^{S} \geq 0 \quad \forall l, i \\
& 0 \leq \bar{g}_{s}^{F}-g_{s}^{F} \perp \beta_{s i}^{S F} \quad \forall s \in \mathfrak{F}, i \\
& 0 \leq g_{S}^{F} \perp \psi_{s i}^{S F} \quad \forall s \in \mathfrak{F}, i \\
& 0 \leq d_{n} \perp \phi_{n i}^{S} \quad \forall n \in \mathfrak{N}, i \\
& 0=\delta_{\hat{n}} \perp \gamma_{i}^{S} \quad \text { (free) } \quad \forall i \\
& 0 \leq \sum_{n \in \mathfrak{N}}\left[\left(a_{n}-\frac{1}{2} b_{n} d_{n}\right) d_{n}\right]-\sum_{s \in \mathfrak{F}} c_{s}^{G} g_{s}^{F}-\frac{1}{2} \sum_{n \in \mathfrak{S}_{n}} \frac{1}{b_{n}}\left[a_{n}-p_{n}+\phi_{n}\right]^{2}+
\end{aligned}
$$




$$
-\sum_{n} p_{n}\left(\sum_{s \in \mathfrak{S}_{n}} g_{s}^{S}\right)-\sum_{l}\left(\bar{f}_{l}+e_{l}\right)\left(\overline{\mu_{l}}+\underline{\mu_{l}}\right)-\sum_{s \in \mathfrak{F}} \beta_{s} \bar{g}_{s}^{F} \perp \xi_{i}^{S} \geq 0 \quad \forall i
$$

One source of non-convexities are the bilinearities in $(6 \mathrm{x})$. Note that we can ignore the complementarity requirement in $\sqrt{6 \mathrm{x}})$ here because the left-hand side cannot be different from zero in any case; otherwise primal or dual feasibility would be violated. Recalling equivalence between this strong duality constraint at hand and the ISO's optimality conditions, we can replace $6 \mathrm{x}$ by the according first order conditions (2a) - 2j). Let us now discuss the two caveats in more detail.

\section{Generalized Nash property: Disjunctive constraints reformulation.}

As we do not want to rely on exogenous assumptions concerning coordination among multiple players, we tackle the GNE problem at hand by a disjunctive constraints reformulation of the complementarity requirements. As an example of how this is implemented, consider conditions (2i), arising from the replacement of the duality constraint $6 \mathrm{x}$ above, and (6v) capturing non-negativity of demand at node $n$ and the associated dual variables.

$$
\begin{array}{lll}
0 \leq d_{n} & \perp \quad \phi_{n} \geq 0 \\
0 \leq d_{n} & \perp & \phi_{n i}^{S} \geq 0 \quad \forall i
\end{array}
$$

In the optimum, either demand is strictly positive with all duals from the ISO's and all strategic generators' perspective zero, or demand is zero with no further requirement except non-negativity for $\phi_{n}$ and all $\phi_{n i}^{S}$. As non-negativity of demand takes effect for both the ISO and all strategic players, there are fewer distinct equations than distinct variables and the emerging system is non-square. We can, however, reformulate the system introducing one binary variable and associated large scalar:

$$
\begin{aligned}
d_{n} \geq 0, \quad \phi_{n} & \geq 0, \quad \phi_{n i}^{S} \geq 0 \quad \forall i \\
d_{n} & \leq r_{n}^{\phi^{S}} K_{n}^{\phi^{S}} \\
\sum_{i} \phi_{n i}^{S}+\phi_{n} & \leq\left(1-r_{n}^{\phi^{S}}\right) K_{n}^{\phi^{S}}
\end{aligned}
$$

Inequalities $7 \mathrm{~d}$ ) and $7 \mathrm{e}$ are linked by the binary variable $r_{n}^{\phi^{S}}$ and scalar $K_{n}^{\phi^{S}}$. There are two possible configurations: either, $r_{n}^{\phi^{S}}$ is zero, forcing the left-hand side of $(7 \mathrm{~d})$ to be non-positive such that demand is zero, or $r_{n}^{\phi^{S}}$ is equals one, preventing the left-hand side of $7 \mathrm{e}$ from positivity such that the sum over all dual variables is zero. Therefore, at least one equality required by conditions (7a), 7b holds, and complementarity condition is implemented for the ISO and all strategic generators. Note that in $7 \mathrm{ee}$, we sum over the dual variable from the ISO's problem and those for all strategic players $i$. This procedure is convenient as it renders a leaner formulation of the KKT conditions without losing information: if demand at a node is zero, then it is zero from the perspective of the ISO and all players.

Applying the same logic to all complementarity requirements attached to inequality constraints leaves us with a complete set of KKT conditions, which can be found in the Appendix. By this means, we formally overcome the inconvenient characteristics of the Generalized Nash property: we do not assign the relative valuation of duals a priori; instead, the duals are chosen such that there is an equilibrium between the strategic generators according to the objective function of the top-level player.

\section{Non-convexity: Algorithmic approach.}

We already noted that neither the constraint set nor the objective function of the strategic players is convex such that both necessity and sufficiency of the KKT conditions cannot 
be guaranteed. Concerning necessity, it may be the case that there are Nash equilibria of the game that do not fulfill the KKT conditions and, hence, are not found by using our methodology. In mathematical terms, those may refer to relaxed definitions of the Nash equilibrium concept such as Nash Bouligand stationarity (Kulkarni and Shanbhag, 2013). We are aware of this gap; however, alternative approaches such as enumerative or GaussSeidel type diagonalization algorithms are also not guaranteed to find all stationary points. In this respect, there is one significant advantage of our approach relative to more ad-hoc approaches: even if we do cannot identify equilibria which are not KKT points: all equilibria that we find are ranked according to the objective function of the first-level player. We can therefore claim that there is no equilibrium satisfying the KKT conditions of the strategic generators, which is better from the point of view of the social planner. In contrast, when one uses genetic algorithms or diagonalization, one can never guarantee that the equilibrium found is not dominated by another solution.

Concerning sufficiency, KKT points do not necessarily describe a Nash equilibrium, but may also constitute saddle points or minima for some strategic players. This issue is much easier to resolve: we implement an iterative algorithm systematically identifying solution candidates among the KKT points. Each of those is checked for deviation incentives for all players, holding the decisions of the network planner and all rivals (strategic generators) fixed. Thereby, we search for global Nash equilibria and are not restricted to analyzing only local deviation incentives (local Nash equilibria, $\mathrm{Hu}$ and Ralph, 2007). By this algorithm, we also account for the potential multiplicity of equilibria. We explain the algorithm in greater detail below.

\section{Sufficiency: Optimistic and pessimistic solutions.}

Sufficiency of a KKT point is linked to incentive compatibility for each player. This, in turn, is affected by an inherent feature of multi-stage equilibrium games: the implicit assumption on optimism versus pessimism.

Consider the set of all solutions to the lower stage (III) - as it is rendered by the ISO optimality conditions in our case. Formally, the decision variables become decision variables on the superordinate stage, here the strategic firms' optimization programs (II). If this set is singleton for each possible strategy of the upper-level players, they do not have any discretion about picking those variables such that it is most profitable to them. If it is not singleton a property that is not guaranteed in our case - then firms will decide on variables such as to maximize their objective. Accordingly, the label optimistic solution applies.

Conversely, it could also be assumed that the lower level player behaves such that it is detrimental for the upper level player(s) in a situation where she is indifferent between several options - for example when she is a rival. Then, the lower level player would have the incentive to behave such that the worst outcome for the upper level players would emerge when they maximize their objective. As the driving forces within such a model pull in opposite directions, it is more elaborate to cover in a mathematically sound manner.

In our formulation, we implicitly pursue the optimistic assumption; as both the lower level ISO and the top-level network planner maximize total welfare, the objectives on these two stages point into the same direction. This becomes especially relevant in the stability discussion for solutions found in our sample network below.

\section{Stage I: Welfare-optimal network expansion}

Equations and inequalities $10 \mathrm{a}-11 \mathrm{l}$ in the Appendix capture all stationary points of the EPEC spot market model. Among those, global and local maxima, minima, and saddle points may be found. The first stage now serves as a selection device, in which a benevolent planner optimizes global welfare deciding on expansion of network capacity (8a). Letting $c_{l}^{E}$ denote the costs, $e_{l}$ the level of network expansion for line $l$, and assembling all dual 
variables of the second-stage game in set $\mathfrak{D}^{2}$, consider her welfare-maximization:

$$
\begin{aligned}
\min _{e, g, d, \delta, \mathfrak{D}^{3}, \mathfrak{D}^{2}} & -\sum_{n \in \mathfrak{N}}\left[\left(a_{n}-\frac{1}{2} b_{n} d_{n}\right) d_{n}\right]+\sum_{s \in \mathfrak{F}} c_{s}^{G} g_{s}^{F}+\sum_{i} \sum_{s \in \mathfrak{S}_{\mathfrak{i}}} c_{s}^{G} g_{s}^{S}+\sum_{l} c_{l}^{E} e_{l} \\
\text { s.t. } \quad & 0 \leq e_{l} \leq \bar{e}_{l} \quad \forall l, \\
& \quad 10 \mathrm{a}
\end{aligned}
$$

where line expansion is bounded between zero and an exogenous maximum $\bar{e}_{l}$ 8b . Program (8a) - 8c can be solved as Mixed-Integer Quadratically-Constrained Quadratic Problem (MIQCQP), which can be solved using solvers such as BARON (Tawarmalani and Sahinidis, 2005) or BONMIN (Bonami et al. 2008).

\section{Iterative algorithm and deviation stability checks.}

To address the issues of sufficiency of the KKT conditions and multiplicity of equilibria, we implement an algorithmic approach systematically exploring the solution space while checking for incentive compatibility. Consider the steps in detail below, where $|\mathfrak{Z}|$ is the exogenously specified number of iterations and $\epsilon$ the difference tolerance level.

1. Find solution candidate. Solve Problem 8 and denote solutions on line expansions by $\hat{e}_{l}(z)$, on generation of strategic firms by $\hat{g}_{s}(z)$ respectively, where $z \in \mathfrak{Z}$ is the index of the iteration.

2. Check for deviation incentives. Fix all variables from the previous solution candidate $z$, except generation choices for strategic player $i$ and re-solve Problem 5 applying the same disjunctive constraints reformulation of complementarity requirements and the same substitution of the duality constraint as above; this is the MPEC of one strategic generator given the network and the rivals' generation. Loop over all strategic players; if one firm $i$ identifies a profitable deviation from the proposed solution candidate $\hat{g}_{s}(z)$, discard this candidate as solution; otherwise, keep it as a Nash equilibrium.

3. Impose difference to previous solutions. Add the following constraints to Problem 8

$$
\begin{aligned}
g_{s} & \geq D_{s}^{g+}(z)\left(\hat{g}_{s}(z)+\epsilon\right) & & \forall s, z \\
g_{s} & \leq \bar{g}_{s}-D_{s}^{g-}(z)\left(\bar{g}_{s}-\hat{g}_{s}(z)+\epsilon\right) & & \forall s, z \\
e_{l} & \geq D_{l}^{e+}(z)\left(\hat{e}_{l}(z)+\epsilon\right) & & \forall l, z \\
e_{l} & \leq \bar{e}_{l}-D_{l}^{e-}(z)\left(\bar{e}_{l}-\hat{e}_{l}(z)+\epsilon\right) & & \forall l, z \\
\sum_{s}\left[D_{s}^{g+}(z)+D_{s}^{g-}(z)\right] & +\sum_{l}\left[D_{l}^{e+}(z)+D_{l}^{e-}(z)\right] \geq 1 & & \forall z
\end{aligned}
$$

where $D(z)$ are binary variables, and $\epsilon$ represents the tolerance distance level for the next solution to be different from all preceding ones. Inequalities $9 \mathrm{a}-9 \mathrm{~d}$ ) implement that no two solution candidates are alike but differ at least by tolerance $\epsilon$ for at least one variable $9 \mathrm{e} \mathrm{H}^{3}$

4. Termination. Repeat procedure until $z=|\mathfrak{Z}|$

The algorithm successively cuts 'holes' into the feasible space and delivers a number of candidate solutions that are ranked according to their welfare objective value. Whether the proposed KKT point indeed constitutes a solution is checked subsequently (step 2); irrespective whether the first, second or third candidate is discarded, this procedure enables us to

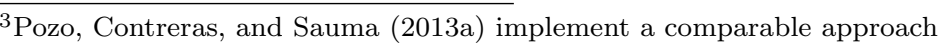


find that equilibrium among the KKT points featuring the highest overall welfare. Alternative solution concepts like diagonalization algorithms crucially depend on starting values and thus convergence to global optimality cannot be guaranteed. One caveat mentioned above, however, persists: non-convexity potentially gives rise to equilibria not fulfilling the KKT conditions, which we cannot identify by our method.

\section{$5 \quad$ Results and Discussion}

We demonstrate the prevailing effects of the trade-off concerning welfare-enhancing, but costly, network expansion when strategic firms are present in a straightforward three-node network. It represents the simplest possible case capturing loop flows. We deliberately choose line capacities such that different strategic effects are present in one network setup. The three nodes are mutually linked by transmission lines with limited capacities of $\bar{f}_{1}=0.5$, $\bar{f}_{2}=1$, and $\bar{f}_{3}=2$. Each line is symmetric insofar as it exhibits the same capacity for flows in both directions. Consumption is located at one node, represented by the linear elastic inverse demand curve $p_{1}=10-q_{1}$, where $q_{1}$ captures the quantity consumed at that node. The other two nodes, at each of which one strategic firm with zero marginal costs is located, feature no consumption. By disregarding potentially asymmetric production costs, we preclude results that are driven by pure efficiency gains: a welfare increase due to substitution effects from expensive to previously inaccessible cheap production could flaw the stylized assessment of reduced market power rents. For convenience, we abstract from competitive fringe generation for now. Consider Figure 1 depicting the network.

Figure 1: Sample network.

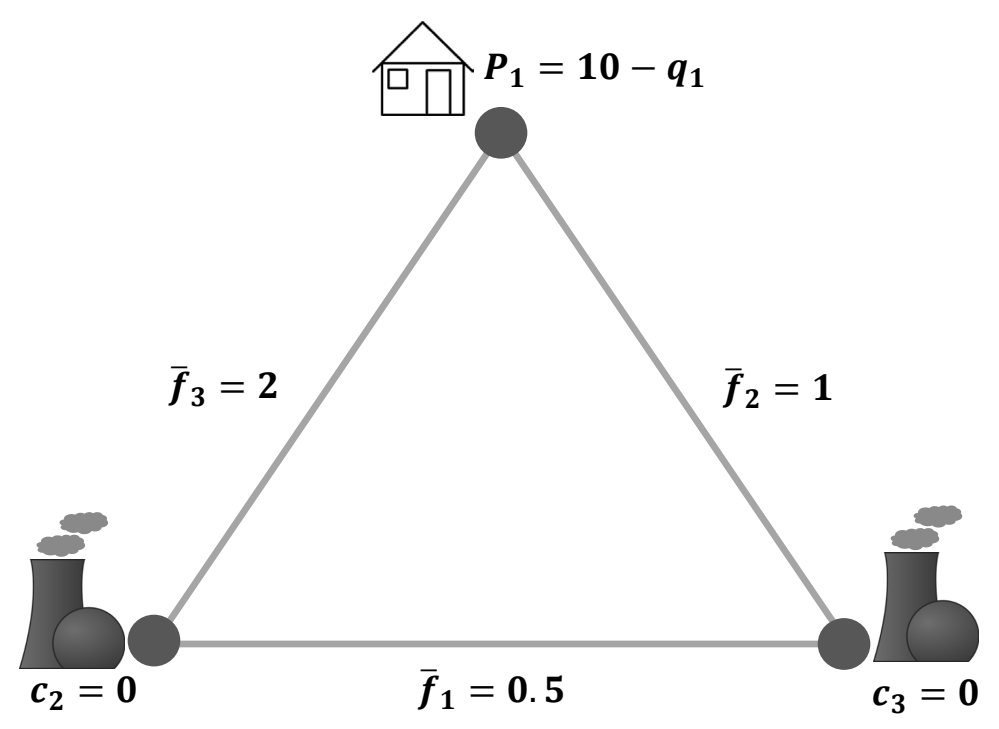

Note: pictograms are under public domain free license.

\section{Benchmark results for strategic firms}

To frame the problem, we first calculate results for two benchmark cases with strategic firms engaging in Cournot competition: Strategic 6 Copperplate, in which all line capacities are set to very large values such that they are never binding; and Strategic 85 No Expansion, in which network expansion is exogenously set to zero. Table 2 presents the numerical results of these two cases. 
The Strategic \& Copperplate result sets an upper welfare benchmark: if line restrictions are never binding, the Cournot solution emerges, representing the maximum degree of competition among the two firms in our setting, unconstrained by any network limitations 4

Table 2: Results for the benchmark cases - strategic firms.

\begin{tabular}{|c|c|c|c|c|}
\hline & & Strategic \& Copperplate & $\begin{array}{l}\text { Strategic } \& 1 \\
\text { highest welfare }\end{array}$ & $\begin{array}{l}\text { o Expansion } \\
\text { lowest welfare }\end{array}$ \\
\hline \multirow{2}{*}{ Generation } & firm 2 & 3.33 & 1.9 & 0 \\
\hline & firm 3 & 3.33 & 0.55 & 1.5 \\
\hline Price & all nodes & 3.33 & 7.55 & 8.5 \\
\hline Demand & node 1 & 6.67 & 2.45 & 1.5 \\
\hline \multirow{3}{*}{ Network flows } & line 1 & 0 & 0.45 & -0.5 \\
\hline & line 2 & 3.33 & 1 & 1 \\
\hline & line 3 & -3.33 & -1.45 & -0.5 \\
\hline Consumer rent & total & 22.22 & 3 & 1.13 \\
\hline \multirow{2}{*}{ Profit } & firm 2 & 11.11 & 14.33 & 0 \\
\hline & firm 3 & 11.11 & 4.16 & 12.75 \\
\hline \multirow{2}{*}{$\begin{array}{c}\text { Congestion rent } \\
\text { Welfare }\end{array}$} & total & 0 & 0 & 0 \\
\hline & total & 44.44 & 21.49 & 13.88 \\
\hline
\end{tabular}

Note: results rounded to two decimals.

The lower benchmarks - or status quo results for strategic players in a given network arise if line upgrades are restricted to zero, see Strategic 85 No Expansion in Table 2 , As there are multiple equilibria, we report the two extreme cases: the one yielding the highest overall welfare, and the one featuring the lowest welfare. We discuss the highest-welfare type first. By being able to produce a greater amount, $g_{2}=1.9$, which can be transported to demand via line $l_{3}$, firm 2 is located in a dominant position and congests the transmission line that connects the passive firm 3 to the demand node. By that strategy, a stable passive-aggressive equilibrium emerges as discussed by Borenstein, Bushnell, and Stoft (2000) in a two-node case. A qualitatively parallel but more extreme configuration emerges in the lowest-welfare Strategic $\&$ No Expansion benchmark. Here, firm 3 behaves aggressively, actively congesting the transmission line that connect the now passive firm 2 to the demand node. Thereby, it drives the rival generator out of the market. Both solutions are stable Nash equilibria in which no firm has an incentive to deviate unilaterally. The mechanism how firms coordinate on one of the equilibria - be it some economic dominance concept, or drivers exogenous to the model like path dependences, policy or regulatory reasons - is beyond the scope of this paper. In any case, from an overall welfare perspective, there is scope for network expansion to enhance welfare.

\section{Investment results}

Let us now turn to the three-stage model featuring welfare-optimal network expansion. Assuming expansion costs of $c_{l}^{E}=1 \forall l$, the benevolent network planner (stage I) selects candidates for optimal solutions; Table 3 presents three distinct types. Note that from this

\footnotetext{
${ }^{4}$ Note that transmission lines are directed in a counter-clockwise fashion. A negative flow, for example -3.33 on line $l_{3}$ in benchmark case Strategic \& Copperplate represents 3.33 units flowing from node 2 to node 1 .
} 
point on, we only aim at finding welfare-optimal equilibria - there may exist more suboptimal solutions to the model similar to the "lowest welfare" equilibrium in the Strategic $\&$ No Expansion case. These are, however, not in our scope of interest, both for modeling reasons and economic interpretation.

Table 3: Results for the network expansion model.

\begin{tabular}{|c|c|c|c|c|}
\hline & & $\begin{array}{c}\text { Myopic } \\
\text { Expansion }(M)\end{array}$ & $\begin{array}{c}\text { Optimal } \\
\text { Expansion } \\
\text { Unstable }(\widetilde{O})\end{array}$ & $\begin{array}{l}\text { Optimal } \\
\text { Expansion } \\
\text { Stable }(O)\end{array}$ \\
\hline \multirow{2}{*}{ Generation } & firm 2 & 1.75 & 3.33 & 3.33 \\
\hline & firm 3 & 2.50 & 3.33 & 3.33 \\
\hline Price & all nodes & 5.75 & 3.33 & 3.33 \\
\hline Demand & node 1 & 4.25 & 6.67 & 6.67 \\
\hline \multirow{6}{*}{$\begin{array}{l}\text { Network capacity } \\
\text { (initial + expansion) }\end{array}$} & \multirow{2}{*}{ line 1} & 0.5 & 0.5 & 0.77 \\
\hline & & $(0.5+\mathbf{0})$ & $(0.5+\mathbf{0})$ & $(0.5+\mathbf{0 . 2 7})$ \\
\hline & \multirow{2}{*}{ line 2} & 2.25 & 3.33 & 3.33 \\
\hline & & $(1+\mathbf{1 . 2 5})$ & $(1+\mathbf{2 . 3 3})$ & $(1+\mathbf{2 . 3 3})$ \\
\hline & \multirow{2}{*}{ line 3} & 2 & 3.33 & 3.33 \\
\hline & & $(2+\mathbf{0})$ & $(2+\mathbf{1 . 3 3})$ & $(2+\mathbf{1 . 3 3})$ \\
\hline \multirow{3}{*}{ Network flows } & line 1 & -0.25 & 0 & 0 \\
\hline & line 2 & 2.25 & 3.33 & 3.33 \\
\hline & line 3 & -2 & -3.33 & -3.33 \\
\hline Consumer rent & total & 9.03 & 22.22 & 22.22 \\
\hline \multirow{2}{*}{ Profit } & firm 2 & 10.07 & 11.11 & 11.11 \\
\hline & firm 3 & 14.36 & 11.11 & 11.11 \\
\hline Congestion rent & total & 0 & 0 & 0 \\
\hline Expansion cost & total & 1.25 & 3.67 & 3.94 \\
\hline Welfare & total & 32.21 & 40.78 & 40.50 \\
\hline
\end{tabular}

Note: results rounded to two decimals.

To begin with, closer inspection of the flow pattern in the Strategic \& No Expansion benchmark case reveals that the only bottleneck in the network present in all cases is line $l_{2}$ - flows on the other lines constrain the dispatch only in some cases. As thus suggested, we firstly restrict expansion to be only possible with regard to this line and myopically constrain upgrades of the other lines $l_{1}$ and $l_{3}$ to zero. The first column of Table 3 renders the result, the type of which we denote by Myopic Expansion ( $M)$ : line $l_{2}$ is expanded by 1.27 units. Compared to the welfare-optimal Strategic \&s No Expansion benchmark, now firm 3 is connected to demand by the "larger" link and enters into the aggressive position whereas firm 2 is pushed into the passive position (relative to the "highest welfare" equilibrium in the Strategic \& No Expansion case). While the firms' roles switch, the quality of this equilibrium is of the same passive-aggressive nature as in the Strategic $\&$ No Expansion benchmark, despite an overall welfare increase from 21.49 to 32.33 . The check for deviation stability indicates incentive compatibility for both strategic players. One interesting implication of this solution, thus, is the following: if network expansion myopically focuses on bottlenecks only, the outcome may be suboptimal in terms of welfare when strategic firms are present; there may be better investment decisions, which are ignored by the network planner due to its focus on the existing bottlenecks. 
In this myopic investment case, only line $l_{2}$ is expanded, because it was the only congested line in all benchmark cases. Although an increase in welfare is achieved by providing relief to that bottleneck, this type of expansion does not prevent strategic firms from gaming the network and extracting rents. The basic mechanism remains unchanged.

Next, let us turn to the Strategic $\&$ Copperplate benchmark. Inspection of the prevailing flows, see Table 2, shows that only lines $l_{2}$ and $l_{3}$ have to be upgraded in order to accommodate this solution. Following this suggestion, we restrict expansion of line $l_{1}$, run the model and arrive at the KKT point Optimal Expansion Unstable $(\widetilde{O})$, see second column of Table 3 . This solution candidate renders the lowest level of line capacities necessary to accommodate the prevailing flows in the Cournot solution, in which competition is never curbed by any congestion. Thus, it would deliver the theoretically highest amount of social welfare that could be attained in this setup. This KKT point, however, turns out to be unstable against deviations of the strategic firms. Therefore, it does not constitute a Nash equilibrium and must be discarded as solution for the model. To illustrate the point, consider the deviation analysis: holding network expansion and firm 3's generation fixed at $g_{3}^{S}=3.33$, and letting firm 2 re-optimize, the MPEC stability check finds a profitable deviation, as summarized in Table 4. Due to symmetry, holding network expansion and firm 2's generation fixed and letting firm 3 re-optimize delivers a parallel result.

Table 4: MPEC stability check: for solution candidate $\widetilde{O}$, firm 2 deviates to 1.83 .

\begin{tabular}{|c|c|c|c|c|c|c|c|c|c|}
\hline & Firm 2 & Firm 3 & $n_{1}$ & $n_{2}$ & $n_{3}$ & $l_{1}$ & $l_{2}$ & $l_{3}$ & Total \\
\hline Generation & 1.83 & 3.33 & & & & & & & \\
\hline Demand & & & 5.17 & 0 & 0 & & & & \\
\hline Price & & & 4.83 & 9.67 & 0 & & & & \\
\hline Network capacity & & & & & & 0.5 & 3.33 & 3.33 & \\
\hline Network flows & & & & & & -0.5 & 2.83 & -2.33 & \\
\hline Consumer rent & & & 13.35 & & & & & & 13.35 \\
\hline Profit & 17.72 & 0 & & & & & & & 17.72 \\
\hline Congestion rent & & & & & & & & & 10.92 \\
\hline Expansion costs & & & & & & 0 & 2.33 & 1.33 & 3.67 \\
\hline Welfare & & & & & & & & & 38.82 \\
\hline
\end{tabular}

Note: results rounded to two decimals.

Firm 2 deviates by lowering its generation to $g_{2}^{S}=1.83$, and increases its profits from 11.11 to 17.72 , while the profits of firm 3 fall to 0 . This outcome is induced by the emerging price pattern, $p_{2}=9.67$ and $p_{3}=0$. The re-optimizing player picks a generation level such that an equilibrium is reached that is most profitable to her.

In order to identify stable solution candidates, we widen the focus on all lines in the network. Result Optimal Expansion Stable (O) in Table 3 reveals that line 1 has to be expanded as well, by $e_{1}=0.27$ to 0.77 . This solution is stable against deviations. Incentive compatibility holds in this equilibrium: line $l_{1}$ now has sufficient capacity such that a unilateral reduction of generation does not evoke a congested equilibrium with substantially differentiated prices, as in $\widetilde{O}$. This is reminiscent of the thin line-effect (Borenstein, Bushnell, and Stoft, 2000): although connection line $l_{1}$ between the two firms does actually not accommodate a positive or negative net flow in equilibrium - as can be seen in Table 3 - its expansion is required to prevent firms from gaming the network and to guarantee stability of the Cournot equilibrium. 


\section{Redistribution of rents}

We now turn to the distributional implications: Figure 2 indicates the outcomes for the three model cases. We first compare the "highest welfare" equilibrium identified in the Strategic 8 No Expansion benchmark case (black bars) with the Myopic Expansion equilibrium (light gray bars). This illustrates that the welfare gain is accompanied by higher producer and consumer rents, where both effects are of comparable size. Observe that the previously aggressive generator (firm 2) is now in the passive position and loses rents; the gain in producer surplus fully accrues for firm 3, which benefits from entering into the aggressive role.

Figure 2: Distribution of rents.

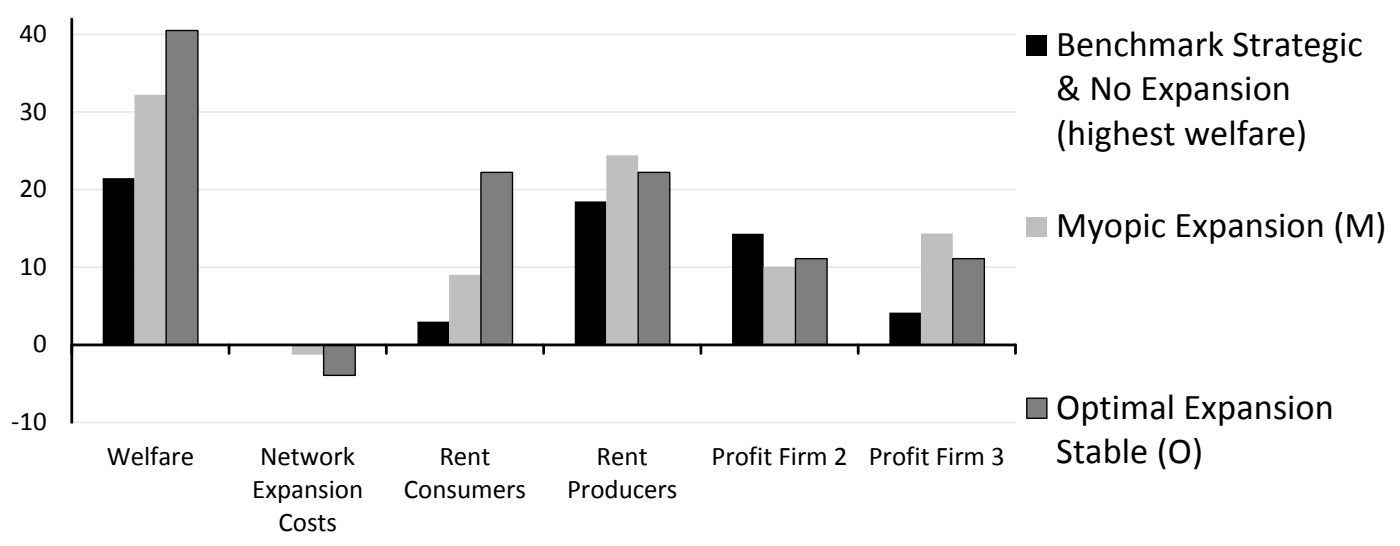

For the stable Cournot equilibrium in the Optimal Expansion case $O$ (dark gray bars), total welfare increases further compared to the Myopic Expansion solution $M$. The distribution of rents, however, reveals that consumer surplus increases whereas total producer surplus decreases. We thus detect a shift of rents from suppliers toward the demand side, mirroring the lowered potential of firms to extract rents due to exploiting a dominant position within the network. Contrasting the Strategic $\&$ No Expansion benchmark directly with the Optimal Expansion equilibrium, the welfare gains accrue for consumers and producers the former, however, profiting to a considerably higher extent, thus entailing a relative shift of rents.

The effect of network expansion on welfare when strategic firms are present, therefore, is twofold: on the one hand, overall rents increase due to the availability of transmission capacity; both consumers and producers benefit. On the other hand, at some point, the network has sufficient capacity to accommodate the Cournot solution, the highest degree of competition in this sample case. Along with the creation of additional rents, there is a redistribution from generators toward consumers - network expansion, thus, has the potential to limit the exertion of market power, and prevents firms from extracting rents that arise due to limited transmission capacity.

\section{Strategic versus competitive firms}

Finally, we want to highlight the impact of modeling firms as strategic players compared to a perfectly competitive market. To this end, we contrast the results of our model with a case in which generators act as price-takers: we remove stage II from our setup, and assign both firms to the competitive fringe being optimized by the ISO while leaving all other parameters unchanged. Table 5 shows the results regarding overall welfare for the No Expansion, Myopic Expansion, and stable Optimal Expansion cases for competitive and 
strategic markets. For illustrative purposes, we include the "lowest welfare" equilibrium in the Strategic \& No Expansion benchmark case in the comparison.

Table 5: Comparison of welfare results for competitive market vs. strategic firms.

\begin{tabular}{cccc} 
& Competitive market & \multicolumn{2}{c}{ Strategic firms } \\
& & highest welfare & lowest welfare \\
\hline \hline No expansion & 21.87 & 21.49 & 13.88 \\
Myopic Expansion $(M)$ & 37.5 & 32.21 & \\
Optimal Expansion $(O)$ & 43.37 & 40.50 & \\
\hline \hline
\end{tabular}

Note: results rounded to two decimals.

Consider first the case when network expansion is suppressed (first row of Table 5): in a competitive market, the ISO dispatches both firms at marginal cost $c_{2}^{G}=c_{3}^{G}=0$, until lines are fully utilized. As the ISO appropriates arbitrage profits, relatively low consumer surplus and high congestion rents emerge. Total welfare amounts to 21.87. The presence of strategic firms, in any case, reduces overall surplus; by ending up in a passive-aggressive equilibrium, they appropriate a certain share of rents. The variance of outcomes in terms of welfare is, however, non-negligible, ranging between 21.49 in the welfare-optimal and 13.88 in the lowest-welfare case. As it is unclear a priori which of these equilibria would be attained and thus the wedge between the competitive and strategic outcomes varies, the detection of market power is non-trivial from a regulatory perspective.

Secondly, consider welfare-optimal network expansion by a benevolent planner (second and third row of Table 5): for a Competitive Market, lines are upgraded as long as the cost for the next unit of expansion would exceed its benefits, yielding increases in overall welfare to 37.5 for Myopic Expansion, and 43.37 for Optimal Expansion, respectively. As discussed above, a qualitatively analogous outcome holds true when generators are assumed strategic; welfare rises to 32.21 , or 40.50 , respectively, at the same time relatively redistributing the additional surplus from producers to consumers.

Finally, examine the relative sizes of the numerical results. If we compare the effect of network expansion on a competitive market with that on a market on which firms behave strategically, two further result emerge: firstly, without expansion the wedge between welfare outcomes can be considerably larger. The presence of strategic firms can lower total welfare up to roughly $60 \%$ (first row, lowest welfare case) if lines cannot be upgraded. If we allow for network expansion, however, welfare on a strategic market is pushed quite close to the competitive benchmark (third row). Network expansion can thus be regarded as a means to approach a first best solution by weakening the position of dominant players. Secondly, myopically focusing on congested lines only can exacerbate the relative welfare-detrimental impact of strategic firm behavior: when analyzing the welfare-optimal market outcomes, without expansion, the wedge between the strategic and the competitive result can be as low as 21.49 compared to 21.87. Under welfare-optimal but myopic line upgrades, this gap widens in absolute and relative terms, compare 32.21 to 37.5 .

We conclude that when designing network expansion, a careful analysis of the status quo has to be carried out whether and to which extent strategic firm behavior is present - and how network expansion may affect gaming opportunities of market participants.

\section{Proactive planning}

These findings also mirror the proactive or anticipative transmission planning paradigm by Sauma and Oren (2006) and Pozo, Contreras, and Sauma (2013a): taking strategic behavior of dominant producers into account alters welfare-optimal expansion decisions. To crosscheck this argument within our framework, we examine the counterfactual: taking Optimal 
Expansion transmission investments arising from the competitive setup as given, we re-run the model for strategic firms without allowing for further line upgrades. The result is that no stable equilibrium can be identified, as strategic firms always find profitable deviations. Assessing the welfare-optimal expansion decisions for a competitive market, this outcome is as expected: investments in lines $l_{2}$ and $l_{3}$ amount to 3.5 units and 2 units, respectively, and create more capacity than necessary to absorb flows in a Cournot equilibrium - 2.33 and 1.33 units would suffice. Although they are not detrimental in terms of evoking additional gaming opportunities, the incurred costs of expansion reduce welfare.

However, as there is no investment into line $l_{1}$, which connects the two strategic generators, its capacity remains too low at 0.5 units to accommodate a stable equilibrium. The same deviation incentives as for the unstable solution candidate $\widetilde{O}$ above emerge: each strategic firm finds it profitable to lower production, evoke congestion on line $l_{1}$ and benefit from the emerging price pattern. Disregarding the presence of strategic players, thus, may yield a network investment pattern triggering disequilibrium outcomes.

In terms of solution concepts, this points at directions for further research: theoretical solutions may be found by extending the Cournot approach to mixed strategies or Edgeworth cycles. Alternatively, other forms of strategic interactions can be analyzed in our three-stage approach like price competition (cf. Dastidar, 1995), supply function equilibria or auctions (cf. Fabra, von der Fehr, and Harbord, 2006).

\section{Computational issues}

In any reformulation using disjunctive constraints, the choice of the large scalars $K$ and the bounds on the variables is fraught with difficulty: choosing a value too small may overly restrict the problem and therefore yield "wrong" solutions (cf. Huppmann, Gabriel, and Leuthold, 2013), while large values may lead to numerical problems. In our three-stage model, this difficulty is exacerbated by the need to provide bounds on the dual variables for the solvers to operate efficiently. In this work, we used both the BARON (Tawarmalani and Sahinidis, 2005) and the BONMIN (Bonami et al., 2008) solvers as implemented in GAMS, and obtained identical results.

To address this issue, we set upper and lower bounds for each dual variable. According to those the required minimum absolute value of the associated large scalars in constraints (11a) - 111) is determined. For example, in eq. $\sqrt{11 g}$

$$
g_{s}^{S} \leq r_{s}^{\psi^{S}} K_{s}^{\psi^{S}}, \quad \psi_{s}^{S} \leq\left(1-r_{s}^{\psi^{S}}\right) K_{s}^{\psi^{S}} \quad \forall s \notin \mathfrak{F}
$$

the large scalar $K_{s}^{\psi^{S}}$ needs to be no greater than $\max \left\{\bar{g}_{s}^{S}, \bar{\psi}_{s}^{S}\right\}$. We conducted an extensive sensitivity analysis and sanity checks on both the bounds for the dual variables and the large scalars for the disjunctive constraints reformulation. We are confident that the bounds and the scalars were chosen sufficiently large so as not to inadvertently restrict the problem.

\section{Conclusions}

Insufficient transmission grid capacities may impede reaping the full benefits of an integrated electricity market. To assess whether and to what extent network expansion promotes social welfare due to enhanced competition above and beyond mere efficiency gains, we set up a three-stage model mimicking the interplay of electricity grid expansion and strategic generation: in the third stage, an ISO sets nodal prices, dispatches competitive fringe plants and ensures feasible flows. Reformulating her KKT conditions with help of strong duality yields the feasible region for the profit-maximization problems of strategic firms engaging in Cournot competition. The first-order KKT conditions of this EPEC capture the set of stationary points for an equilibrium of the spot market game. To choose the welfare-optimal 
outcome out of these, a benevolent planner forms the first stage (top-level) of the model. She maximizes total welfare by deciding on network expansion. To ensure that the KKT points found are indeed equilibria of the spot market game (i.e., incentive compatibility holds for each strategic generator), we implement an algorithm exploring the solution space in an iterative procedure, and check deviation incentives for each strategic player.

Numerical findings using a three-node network illustrate the strategic effects. Three major results emerge: firstly, by mitigating market power, network expansion has the potential to enhance welfare beyond efficiency gains. Firms are prevented from ending up in equilibria where they deliberately congest transmission lines to appropriate excess rents; consequently, competition is fostered. Myopically focusing on investment options in congested lines only does not guarantee evoking the full extent of competition and may leave leeway for firms to game the network. Comparisons to competitive benchmarks further reveal that network expansion can push welfare considerably closer to a first-best solution.

Secondly, transmission grid expansion, when preventing asymmetric passive-aggressive equilibria as described by Borenstein, Bushnell, and Stoft (2000), can induce a relative shift of rents from generation toward the demand side. Thus, the overall welfare gains coincide with a redistribution of the economic surplus.

Thirdly, spuriously neglecting firms' strategic behavior yields a misjudgment of investment needs regarding transmission: while some lines are upgraded at great costs beyond need, others are not expanded to the level required for a stable solution; this may trigger disequilibrium outcomes.

We contribute to the literature by developing a model that endogenizes the trade-off between welfare-beneficial effects of network expansion when strategic generators are present; they anticipate their impact on the transmission grid operation. By application of recent advances in solving EPEC games, we do not have to rely on exogenous variations in relevant parameters or neglect the strategic interactions between generators and network operation.

The model is, so far, limited to analyzing static one-shot games. Dynamic effects from supergame strategies could uphold equilibria benefiting dominant firms, although network capacities would accommodate more intense competition (cf. Gebhardt and Höffler, 2013). We do not capture varying resistance parameters arising from a changed network topology, and line expansions are decided by a benevolent planner maximizing overall welfare. The strategic dimension thereof, for example when national governments or regulators are in charge, is left open in this contribution.

This paper opens several avenues for future research: for instance, an application of the model to a representation of the European electricity system, based on the aggregation of realistic data, will be able to assess benefits of further electricity market integration in terms of the welfare-enhancing effects of more vigorous competition. A further elaboration on the distributional implications can, moreover, allow for an analysis of gains and losses from integration, which is, in turn, connected to the question of incentive compatibility when it comes to integration among sovereign states pursuing own agendas. Nevertheless, we provide a novel framework that delivers insights on strategic effects for competition in constraining networks.

\section{References}

ACER/CEER. Annual Report on the Results of Monitoring the Internal Electricity and Natural Gas Markets in 2012, 2013.

V. Böckers, J. Haucap, and U. Heimeshoff. Benefits of an integrated European electricity market. DICE Discussion Paper, 109, 2013.

F. Boffa, V. Pingali, and D. Vanconi. Increasing market interconnection: An analysis of 
the Italian electricity spot market. International Journal of Industrial Organization, 28: $311-322,2010$.

P. Bonami, L.T. Biegler, A.R. Conn, G. Cornuéjols, I.E. Grossmann, C.D. Laird, J. Lee, A. Lodi, F. Margot, N. Sawaya, and A. Wächter. An algorithmic framework for convex mixed integer nonlinear programs. Discrete Optimization, 5:186-204, 2008.

S. Borenstein, J. Bushnell, and S. Stoft. The competitive effects of transmission capacity in a deregulated electricity industry. The RAND Journal of Economics, 31(2):294-325, Summer 2000.

L.B. Cunningham, R. Baldick, and M.L. Baughman. An empirical study of applied game theory: Transmission constrained Cournot behavior. IEEE Transactions on Power Systems, 17(1):166-172, 2002.

K.G. Dastidar. On the existence of pure strategy Bertrand equilibrium. Economic Theory, 5:19-32, 1995.

A. Ehrenmann and K. Neuhoff. A comparison of electricity market designs in networks. Operations Research, 57(2):274-286, 2009.

European Commission. Energy, transport and environment Indicators 2012 edition. Publications Office of the European Union, 2012.

N. Fabra, N.-H. von der Fehr, and D. Harbord. Designing electricity auctions. RAND Journal of Economics, 37(1):23-46, 2006.

J. Fortuny-Amat and B. McCarl. A representation and economic interpretation of a two-level programming problem. The Journal of the Operational Research Society, 32(9):783-792, 1981.

S.A. Gabriel and F.U. Leuthold. Solving discretely-constrained MPEC problems with applications in electric power markets. Energy Economics, 32:3-14, 2010.

S.A. Gabriel, S.A. Siddiqui, A.J. Conejo, and C. Ruiz. Solving discretely-constrained NashCournot games with an application to power markets. Networks and Spatial Economics, 13(3):307-326, 2013.

G. Gebhardt and F. Höffler. How competitive is cross-border trade of electricity? Theory and evidence from European electricity markets. The Energy Journal, 34(1):125-154, 2013.

P.T. Harker. Generalized Nash games and quasi-variational inequalities. European Journal of Operational Research, 54(1):81-94, 1991.

B.F. Hobbs and F.A.M. Rijkers. Strategic generation with conjectured transmission price responses in a mixed transmission pricing system-Part I: Formulation. IEEE Transactions on Power Systems, 19(2):707-717, 2004.

B.F. Hobbs, C.B. Metzler, and J.-S. Pang. Strategic gaming analysis for electric power systems: An MPEC approach. IEEE Transactions on Power Systems, 15(2):638-645, 2000 .

B.F. Hobbs, F.A.M. Rijkers, and M.G. Boots. The more cooperation, the more competition? A Cournot anaylsis of the benefits of electric market coupling. The Energy Journal, 26 (4):69-98, 2005 .

$\mathrm{X}$. Hu and D. Ralph. Using EPECs to model bilevel games in restructured electricity markets with locational prices. Operations Research, 55(5):809-827, 2007. 
D. Huppmann and J. Egerer. National-strategic investment in European cross-border transmission capacity. DIW Discussion Paper, 1379, 2014.

D. Huppmann, S.A. Gabriel, and F.U. Leuthold. A note on allowing negative energy prices in a discretely constrained MPEC. Energy Economics, 40:1023-1025, 2013.

A.A. Kulkarni and U.V. Shanbhag. A shared-constraint approach to multi-leader multifollower games. Working Paper, 2013.

F. Kunz and A. Zerrahn. The benefit of coordinating congestion management in Germany. DIW Discussion Paper, 1298, 2013.

Th.-O. Léautier. Fred Schweppe meets Marcel Boiteux and Antoine-Augustin Cournot: Transmission constraints and strategic underinvestment in electric power generation. Toulouse School of Economics Working Paper, 432, 2013.

F.U. Leuthold, H. Weigt, and C. v. Hirschhausen. A large-scale spatial optimization model of the European electricity market. Networks and Spatial Economics, 12(1):75-107, 2012.

T. Limpaitoon, Y. Chen, and S.S. Oren. The impact of imperfect competition in emission permits trading on oligopolistic electricity markets. The Energy Journal, 35(3):145-166, 2014.

K. Neuhoff, J. Barquin, M.G. Boots, A. Ehrenmann, B.F. Hobbs, F.A.M. Rijkers, and M. Vazquez. Network-constrained Cournot models of liberalized electricity markets: The devil is in the details. Energy Economics, 27:495-525, 2005.

G. Oggioni, Y. Smers, E. Allevi, and S. Schaible. A generalized Nash equilibrium model of market coupling in the European power system. Networks and Spatial Economics, 12(4): 503-560, 2012.

D. Pozo, J. Contreras, and E. Sauma. If you build it, he will come: Anticipative power transmission planning. Energy Economics, 36:135-146, 2013a.

D. Pozo, E.E. Sauma, and J. Contreras. A three-level static MILP model for generation and transmission expansion planning. IEEE Transactions on Power Systems, 28(1):202-210, 2013b.

D. Ruderer and G. Zöttl. The impact of transmission pricing in network industries. EPRG Working Paper 1214, 2012.

C. Ruiz, A.J. Conejo, and Y. Smeers. Equilibria in an oligopolistic electricity pool with stepwise offer curves. IEEE Transactions on Power Sytems, 27(2):752-761, 2012.

N. Ryan. The competitive effects of transmission infrastructure in the Indian electricity market. Working Paper, 2013.

E.E. Sauma and S.S. Oren. Proactive planning and valuation of transmission investments in restructured electricity markets. Jorunal of Regulatory Economics, 30:358-387, 2006.

F.C. Schweppe, M.C. Caramanis, R.D. Tabors, and R.E. Bohn. Spot Pricing of Electricity. Kluwer, Boston, 1988.

S. Siddiqui and S.A. Gabriel. An SOS1-based approach for solving MPECs with a natural gas market application. Networks and Spatial Economics, 13(2):205-227, 2013.

M. Tanaka. Transmission-constrained oligopoly in the Japanese electricity market. Energy Economics, 31:690-701, 2009. 
M. Tawarmalani and N.V. Sahinidis. A polyhedral branch-and-cut approach to global optimization. Mathematical Programming, 103(2):225-249, 2005.

B. Willems. Modeling Cournot competition in an electricity market with transmission constraints. The Energy Journal, 23(3):95-125, 2002.

B. Willems, I. Rumiantseva, and H. Weigt. Cournot versus supply functions: What does the data tell us? Energy Economics, 31:38-47, 2009.

F.A. Wolak. Measuring the competitive benefits of a transmission investment policy: The case of the Alberta electricity market. Working Paper, 2012.

G. Zachmann. Electricity wholesale market prices in Europe: Convergence? Energy Economics, 30:1659-1671, 2008.

G. Zöttl. On optimal scarcity prices. International Journal of Industrial Organization, 29: 589-605, 2011.

\section{A Appendix}

\section{A.1 KKT conditions of the strategic players}

The following equations and inequalities constitute the set of KKT conditions for all strategic players. Each vector fulfilling (10a) - 111) constitutes a stationary point of the spot market equilibrium problem. First all KKT conditions without complementarity requirements.

$$
\begin{aligned}
& -p_{n, s \in \mathfrak{S}_{n}}+c_{s}^{G}+\beta_{s}^{S}-\psi_{s}^{S}-\iota_{n i, s \in \mathfrak{S}_{n}, s \in \mathfrak{S}_{i}}^{S}+\xi_{i, s \in \mathfrak{S}_{i}}^{S} p_{n, s \in \mathfrak{S}_{n}}=0 \quad \forall s \notin \mathfrak{F} \\
& -\iota_{n i, s \in \mathfrak{S}_{n}}^{S}+\beta_{s i}^{S F}-\psi_{s i}^{S F}+\xi_{i}^{S}\left(c_{s}^{G}+\beta_{s}\right)=0 \quad \forall s \in \mathfrak{F}, i \\
& -\sum_{s \in\left(\mathfrak{S}_{n} \cap \mathfrak{S}_{i}\right)} g_{s}^{S}-\sum_{s \in\left(\mathfrak{S}_{n} \cap \mathfrak{F}\right)} \zeta_{s i}^{S}+\eta_{n i}^{S}+\sum_{k} \theta_{k i}^{S} B_{n k}+ \\
& +\xi_{i}^{S} \frac{1}{b_{n}}\left(p_{n}-a_{n}-\phi_{n}\right)+\xi_{i}^{S} \sum_{s \in \mathfrak{S}_{n}} g_{s}^{S}=0 \quad \forall n, i \\
& b_{n} \eta_{n i}^{S}+\iota_{n i}^{S}-\phi_{n i}^{S}-\xi_{i}^{S}\left(a_{n}-b_{n} d_{n}\right)=0 \quad \forall n, i \\
& \sum_{k} B_{k n} \iota_{k i}^{S}+\sum_{l} H_{l n}\left(\bar{\mu}_{l i}^{S}-\underline{\mu}_{l i}^{S}\right)+\left\{\begin{array}{cl}
\gamma_{i}^{S} & \text { if } n=\hat{n} \\
0 & \text { else }
\end{array}\right\}=0 \quad \forall n, i \\
& \zeta_{s i}^{S}+\xi_{i}^{S} \bar{g}_{s}^{F} \geq 0 \quad \forall s \in \mathfrak{F}, i \\
& -\zeta_{s i}^{S} \geq 0 \quad \forall s \in \mathfrak{F}, i \\
& -\eta_{n i}^{S}+\xi_{i}^{S}\left[\frac{1}{b_{n}}\left(\phi_{n}+a_{n}-p_{n}\right)\right] \geq 0 \quad \forall n, i \\
& \sum_{n} \theta_{n i}^{S} H_{l n}+\xi_{i}^{S}\left(\bar{f}_{l}+e_{l}\right) \geq 0 \quad \forall l, i \\
& -\sum_{n} \theta_{n i}^{S} H_{l n}+\xi_{i}^{S}\left(\bar{f}_{l}+e_{l}\right) \geq 0 \quad \forall l, i \\
& \theta_{\hat{n} i}^{S}=0 \quad \forall i \\
& \bar{g}_{s}^{S}-g_{s}^{S} \geq 0 \quad \forall s \notin \mathfrak{F} \\
& g_{s}^{S} \geq 0 \quad \forall s \notin \mathfrak{F} \\
& c_{s}^{G}-p_{n, s \in \mathfrak{S}_{n}}+\beta_{s}-\psi_{s}=0 \quad \forall s \in \mathfrak{F}, i \\
& -a_{n}+b_{n} d_{n}+p_{n}-\phi_{n}=0 \quad \forall n \in \mathfrak{N}, i \\
& \sum_{k} B_{k n} p_{k}+\sum_{l} H_{l n}\left(\bar{\mu}_{l}-\underline{\mu}_{l}\right)+\left\{\begin{array}{ll}
\gamma & \text { if } n=\hat{n} \\
0 & \text { else }
\end{array}\right\}=0 \quad \forall n, i \\
& -\sum_{s \in \mathfrak{S}_{n}}\left(g_{s}^{S}+g_{s}^{F}\right)+\sum_{k} B_{n k} \delta_{k}+d_{n}=0 \quad \forall n, i
\end{aligned}
$$




$$
\begin{aligned}
\bar{f}_{l}+e_{l}-\sum_{k} H_{l k} \delta_{k} \geq 0 & \forall l, i \\
\bar{f}_{l}+e_{l}+\sum_{k} H_{l k} \delta_{k} \geq 0 & \forall l, i \\
\bar{g}_{s}^{F}-g_{s}^{F} \geq 0 & \forall s \in \mathfrak{F}, i \\
g_{S}^{F} \geq 0 & \forall s \in \mathfrak{F}, i \\
d_{n} \geq 0 & \forall n, i \\
\delta_{\hat{n}}=0 & \forall i
\end{aligned}
$$

Next, we list all complementarity requirements which are replaced by disjunctive constraints.

$$
\begin{aligned}
& \sum_{i}\left(\zeta_{s i}^{S}+\xi_{i}^{S} \bar{g}_{s}^{F}\right) \leq r_{s}^{\beta} K_{s}^{\beta}, \quad \beta_{s} \leq\left(1-r_{s}^{\beta}\right) K_{s}^{\beta} \quad \forall s \in \mathfrak{F} \\
& \sum_{i}\left(-\zeta_{s i}^{S}\right) \leq r_{s}^{\psi} K_{s}^{\psi}, \quad \psi_{s} \leq\left(1-r_{s}^{\psi}\right) K_{s}^{\psi} \quad \forall s \in \mathfrak{F} \\
& \sum_{i}\left(-\eta_{n i}^{S}+\xi_{i}^{S}\left[\frac{1}{b_{n}}\left(\phi_{n}+a_{n}-p_{n}\right)\right]\right) \leq r_{n}^{\phi} K_{n}^{\phi}, \quad \phi_{n} \leq\left(1-r_{n}^{\phi}\right) K_{n}^{\phi} \quad \forall n \\
& \sum_{i}\left(\sum_{n} \theta_{n i}^{S} H_{l n}+\xi_{i}^{S}\left(\bar{f}_{l}+e_{l}\right)\right) \leq r_{l}^{\bar{\mu}} K_{l}^{\bar{\mu}}, \quad \bar{\mu}_{l} \leq\left(1-r_{l}^{\bar{\mu}}\right) K_{l}^{\bar{\mu}} \quad \forall l \\
& \sum_{i}\left(\sum_{n} \theta_{n i}^{S} H_{l n}+\xi_{i}^{S}\left(\bar{f}_{l}+e_{l}\right)\right) \leq r \frac{\mu}{l} K_{l}^{\underline{\mu}}, \quad \quad \underline{\mu}_{l} \leq\left(1-r_{l}^{\underline{\mu}}\right) K_{l}^{\underline{\mu}} \quad \forall l \\
& \bar{g}_{s}^{S}-g_{s}^{S} \leq r_{s}^{\beta^{S}} K_{s}^{\beta^{S}}, \quad \beta_{s}^{S} \leq\left(1-r_{s}^{\beta^{S}}\right) K_{s}^{\beta^{S}} \quad \forall s \notin \mathfrak{F} \\
& g_{s}^{S} \leq r_{s}^{\psi^{S}} K_{s}^{\psi^{S}}, \quad \psi_{s}^{S} \leq\left(1-r_{s}^{\psi^{S}}\right) K_{s}^{\psi^{S}} \quad \forall s \notin \mathfrak{F} \\
& \bar{f}_{l}+e_{l}-\sum_{k} H_{l k} \delta_{k} \leq r_{l}^{\bar{\mu}^{S}} K_{l}^{\bar{\mu}^{S}}, \quad \quad \sum_{i} \bar{\mu}_{l i}^{S}+\bar{\mu}_{l} \leq\left(1-r_{l}^{\bar{\mu}^{S}}\right) K_{l}^{\bar{\mu}^{S}} \quad \forall l \\
& \bar{f}_{l}+e_{l}-\sum_{k} H_{l k} \delta_{k} \leq r_{l}^{\underline{\mu}^{S}} K_{l}^{\underline{\mu}^{S}}, \quad \sum_{i} \underline{\mu}_{l i}^{S}+\underline{\mu}_{l} \leq\left(1-r_{l}^{\underline{\mu}^{S}}\right) K_{l}^{\underline{\mu}^{S}} \quad \forall l \\
& \bar{g}_{s}^{F}-g_{s}^{F} \leq r_{s}^{\beta^{S F}} K_{s}^{\beta^{S F}}, \quad \sum_{i} \beta_{s i}^{S F}+\beta_{s} \leq\left(1-r_{s}^{\beta^{S F}}\right) K_{s}^{\beta^{S F}} \quad \forall s \in \mathfrak{w} \\
& g_{s}^{F} \leq r_{s}^{\psi^{S F}} K_{s}^{\psi^{S F}}, \quad \sum_{i} \psi_{s i}^{S F}+\psi_{s} \leq\left(1-r_{s}^{\psi^{S F}}\right) K_{s}^{\psi^{S F}} \quad \forall s \in \mathfrak{F} \\
& d_{n} \leq r_{n}^{\phi^{S}} K_{n}^{\phi^{S}}, \quad \sum_{i} \phi_{n i}^{S}+\phi_{n} \leq\left(1-r_{n}^{\phi^{S}}\right) K_{n}^{\phi^{S}} \quad \forall n
\end{aligned}
$$

